\title{
Einsatz eines Digitalen Zwillings zur Prozessoptimierung und prädiktiven Instandhaltung
} Am Beispiel von Werkzeugmaschinen

\author{
Benedikt Schmucker, \\ Johannes Ellinger, \\ Maximilian Benker, \\ Thomas Semm und \\ Michael F. Zäh, München
}

\section{| Einleitung}

Die Produktivität von Werkzeugmaschinen kann durch eine Erhöhung des Zeitspanvolumens und somit durch eine Reduktion der Hauptzeiten gesteigert werden. Infolgedessen ist jedoch auch ein verstärkter Werkzeug- und Anlagenverschleiß durch höhere Prozesskräfte zu beobachten. Die Prozessregelung und -optimierung muss deshalb sicherstellen, dass trotz des Wunsches nach kurzen Zykluszeiten sowohl die Stabilitätsgrenzen der spanenden Bearbeitung eingehalten als auch die maximalen Maschinenund Werkzeuglasten nicht überschritten werden [1].

Neben der sinkenden Werkzeug- und Anlagenstandzeit nimmt in der Regel auch die Fertigungsgenauigkeit mit stei-

\footnotetext{
*) Danksagung

Die dem Bericht zugrunde liegenden Forschungsvorhaben „CLM 4.0“ und „PreCoM“ werden mit Mitteln des Bundesministeriums für Bildung und Forschung (Förderkennzeichen: 01DM19010A) und der Europäischen Kommission (Grant Agreement No. 768575) gefördert. Die Verantwortung für den Inhalt dieser Veröffentlichung liegt bei den Autoren.
}

Der wirtschaftliche Einsatz von Werkzeugmaschinen ist maßgeblich abhängig von dem erreichbaren Zeitspanvolumen sowie den Stillstandszeiten aufgrund von Wartungsmaßnahmen. Es ist folglich der stete Wunsch von produzierenden Unternehmen, die Belegungszeit und die Anzahl notwendiger Instandhaltungsmaßnahmen zu reduzieren. Der Digitale Zwilling bietet durch einen permanenten Datenaustausch zwischen der realen Werkzeugmaschine und deren virtueller Repräsentation die Möglichkeit, Bearbeitungsprozesse unter Einhaltung der statischen und dynamischen Lastgrenzen zu optimieren und die Restlebensdauer von Maschinenkomponenten zu prognostizieren. *)

genden Schnitttiefen und Vorschubgeschwindigkeiten ab, da höhere Prozesskräfte größere Formfehler verursachen und bei Überschreiten der Grenzschnitttiefe Rattermarken auf der bearbeiteten Oberfläche zurückbleiben [2].

Das Ziel des kanadisch-deutschen Forschungsprojekts „Closed-Loop-Manufacturing 4.0“ (kurz: „CLM 4.0“) ist es, dynamische Instabilitäten durch die virtuelle Abbildung des Bearbeitungsprozesses zu vermeiden und die Vorschubgeschwindigkeit im Hinblick auf die Maschinenbelastung und Formfehler zu optimieren. Hierfür ist es unerlässlich, das positionsund werkzeugabhängige Nachgiebigkeitsverhalten der Maschine zu bestimmen. Eine Online-Modalanalyse erlaubt es, die Werkzeug- und Maschinendynamik während der Bearbeitung zu messen und über den gesamten Lebenszyklus der Anlage zu aktualisieren.

Neben geringen Zeitspanvolumina verursachen ungeplante und lange Stillstandszeiten in immer stärker vernetzten Produktionslinien hohe Kosten. Die sogenannte prädiktive Instandhaltung ermög-

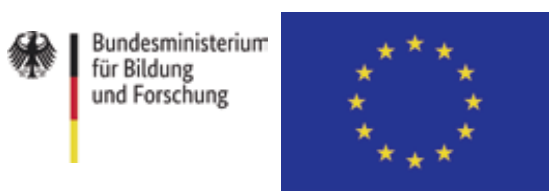

licht es, auf Basis einer umfangreichen Überwachung des aktuellen Maschinenzustandes den Ausfallzeitpunkt verschiedener Anlagenkomponenten vorherzusagen. Aufgrund dessen können Wartungsmaßnahmen in der Produktionsplanung berücksichtigt und ungeplante, kostenintensive Fertigungsunterbrechungen reduziert werden [3]. Die besonders ausfallkritischen Baugruppen von Werkzeugmaschinen sind die in den Vorschubachsen verbauten Kugelgewindetriebe und Profilschienenführungen. Das Forschungsvorhaben „PreCoM“ („Predictive Cognitive Maintenance Decision Support System“) widmet sich deshalb einem intelligenten Ansatz zur Zustandsüberwachung dieser Komponenten, der auf einer Bestimmung des dynamischen Verhaltens der Vorschubachsen basiert [4].

Im nächsten Kapitel wird vorgestellt, wie eine effiziente Akquisition von Maschinen- und Sensordaten eine OnlineProzessregelung und eine Identifikationen der Maschinendynamik während der Bearbeitung ermöglicht. Auch der vom $i w b$ verfolgte Ansatz zur Zustandsüberwachung der Vorschubkomponenten basiert auf einer Identifikation des dynamischen Verhaltens. Die Gestaltung eines hierfür geeigneten Testzyklus und die dazugehörige Datenverarbeitung werden im darauf folgenden Kapitel beleuchtet. 


\section{Edge- und cloudbasierte Prozessoptimierung}

Effiziente Akquisition und Verarbeitung von Maschinendaten durch eine kaskadierte Systemarchitektur

Für die virtuelle Planung, die Echtzeitüberwachung und die Optimierung von spanenden Fertigungsprozessen sind mathematische Modelle der eingesetzten Werkzeugmaschine und des Bearbeitungsprozesses unerlässlich. Je detaillierter die Maschinendynamik und der Bearbeitungsprozess abgebildet sind, desto genauer können statische Formfehler und dynamische Instabilitäten vorhergesagt werden. Allerdings steigt mit dem Detaillierungsgrad der Prozess- und Anlagenmodellierung auch der damit verbundene Rechenaufwand [5]. Aufgrund dessen gilt es zum einen, die Komplexität der eingesetzten physikalischen Simulationsmodelle auf ein notwendiges Niveau zu reduzieren, damit durch die Auslastung der Recheneinheiten die Auflösung der aufgezeichneten Messwerte nicht sinkt und Stellgrößen die Anlagensteuerung rechtzeitig erreichen. Zum anderen ermöglicht die effiziente Systemarchitektur des Forschungsprojektes „CLM 4.0“, diesen Zielkonflikt zu beherrschen. Diese zeichnet sich durch eine Aufteilung in echtzeitkritische Regelungsaufgaben und in rechenintensive Optimierungsschritte aus (Bild 2).

Eine gemeinsame Schnittstelle, ein sogenannter Edge-Computer, stellt den beiden Regelkreisen die steuerungsinternen Maschinendaten und die externen Sensorsignale gepuffert und in einer zeitlich hohen Auflösung für die Weiterverarbeitung zur Verfügung. Der innere Regelkreis arbeitet echtzeitnah und übernimmt alle Aufgaben, die eine schnelle Stellsignal-Rückführung benötigen. Hierzu zählen beispielsweise eine Rattervermeidung, eine Kollisionsdetektion und eine Werkzeugverschleißüberwachung. Die rechenaufwendigeren Systemidentifikationsalgorithmen zur Bestimmung des positions- und werkzeugabhängigen Nachgiebigkeitsverhaltens der Werkzeugmaschine werden hingegen in dem äußeren Regelkreis implementiert.

\section{Rattererkennung und -vermeidung} In Abhängigkeit der Maschinennachgiebigkeit kann bei Überschreiten der Grenzschnitttiefe sogenanntes regeneratives Rattern auftreten. Diese dynamische Instabilität reduziert durch relative Schwingungen zwischen Werkzeug und Werk-

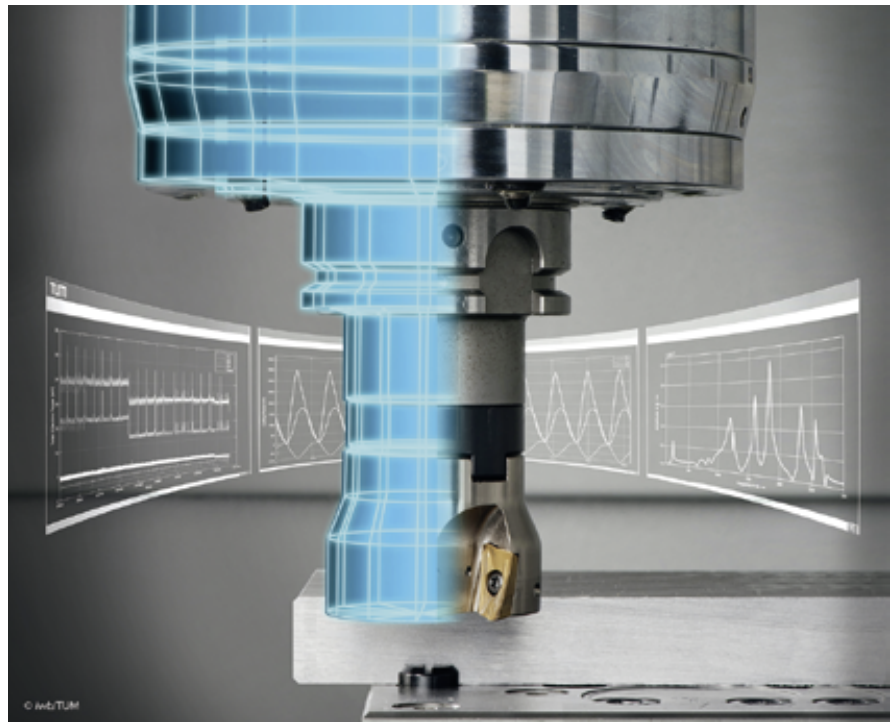

stück und damit durch eine Spanungsdickenmodulation die Oberflächenqualität des finalen Bauteils. Neben dem Zurückbleiben von Rattermarken erhöht sich durch diese ungewollten Schwingungen auch die Belastung am Werkzeug und an Vorschubantriebskomponenten. Dabei verstärken besonders die durch die variierende Spanungsdicke verursachten Wechsellasten den Verschleiß an diesen Komponenten [2]. Regeneratives Rattern wird den selbsterregten Schwingungen zugeordnet. Im Falle einer Selbsterregung schwingt das Maschinensystem in einer oder mehreren Eigenfrequenzen, und es sind dabei meist große Schwingungsamplituden zu beobachten. Aufgrund dessen wird die Zerspanungsleistung bei der Fräs- und Drehbearbeitung häufig nicht durch die installierte Motorleistung, sondern durch das Nachgiebigkeitsverhalten und somit die Ratterneigung begrenzt.

Um zudem hohen Verschleiß am Werkzeug und an der Anlage zu verhindern, muss regeneratives Rattern während der Bearbeitung rasch erkannt und vermieden werden [6]. Die besondere Herausforderung ist dabei, prozessbedingte fremderregte Vibrationen, die unter anderem durch den periodischen Zahneingriff verursacht werden, von Ratterfrequenzen $\mathrm{zu}$ unterscheiden. Charakteristisch für den Regenerativeffekt ist jedoch die Tatsache, dass sich die Schwingungsfrequenz mit Variation der Spindeldrehzahl kaum verändert [2].

In der Literatur existieren verschiedene Verfahren für die Rattererkennung. Zum einen kann diese Sonderform der selbsterregten Schwingungen mit externer Sensorik, wie zum Beispiel Beschleunigungssensoren oder Mikrofonen [6], oder zum anderen auch über die Beobachtung des Spindelmotorstroms detektiert werden [7].

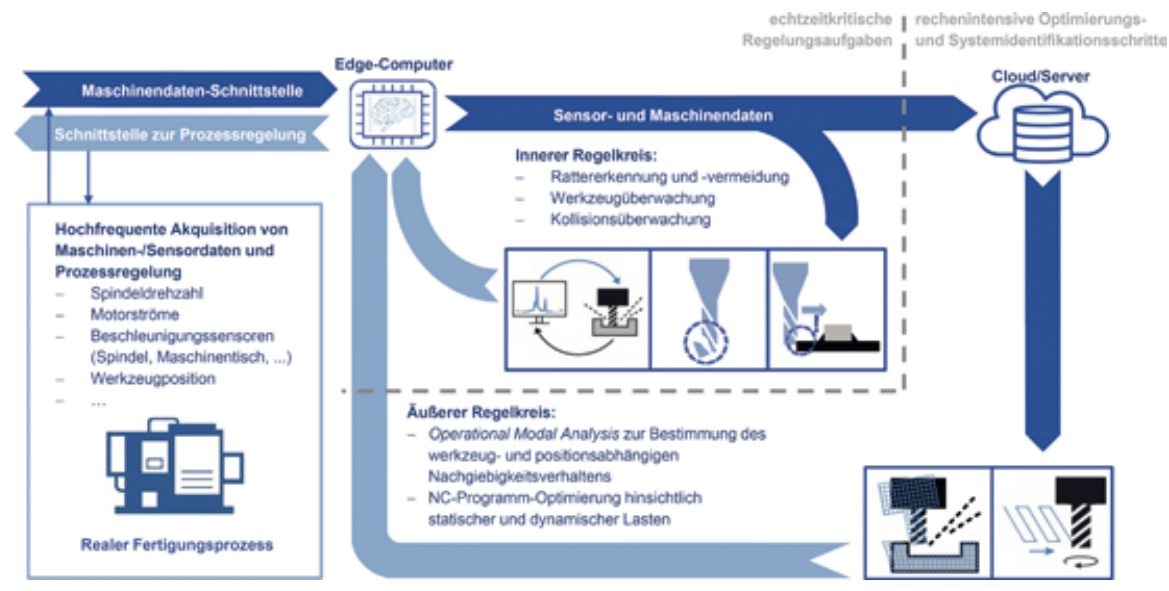

Bild 2. Systemarchitektur zur kaskadierten Prozessregelung und Systemidentifikation des Forschungsprojekts „Closed-Loop-Manufacturing 4.0“ 
Sobald regeneratives Rattern während der Bearbeitung festgestellt wird, gilt es, dieses aufgrund der negativen Einflüsse auf die Bauteilqualität und die Werkzeugund Maschinenstandzeit unmittelbar abzustellen. Zur Rattervermeidung stehen neben konstruktiven Maßnahmen, wie einer Reduktion der Maschinennachgiebigkeit, auch verschiedene regelungstechnische Ansätze zur Verfügung. Das Forschungsvorhaben „CLM 4.0“ zieht Anpassungen der Maschinenkonstruktion und den Einsatz von aktiven Schwingungsdämpfern wegen der hohen Kosten für Nachrüstungen und der zusätzlichen Störkonturen im Arbeitsraum jedoch nicht in Betracht [8]. Stattdessen fokussiert sich das kanadisch-deutsche Verbundprojekt auf die Regelungsstrategien „Discrete Spindle Speed Tuning“ (DSST) (dt. Diskrete Anpassung der Spindeldrehzahl) und „Continuous Spindle Speed Variation“ (CSSV) (dt. Periodische Drehzahlvariation) [9].

Sowohl DSST als auch CSSV können mit sogenannten Stabilitätskarten erklärt werden. Diese stellen in Abhängigkeit der Schnitttiefe $a_{p}$ und der Spindeldrehzahl $n$ die stabilen und instabilen Parameterkombinationen dar. Für die Bestimmung der stabilen Fertigungsbedingungen sind folgende Informationen erforderlich:

- Zerspankraftkoeffizienten,

- Nachgiebigkeitsfrequenzgang von Werkzeug und Werkstück (absolut oder relativ zwischen den beiden Komponenten),

v gewählte Prozessparameter (Schnitttiefe $a_{p}$, Vorschubgeschwindigkeit $f$, Spindeldrehzahl $n$ ) sowie

- Werkzeuggeometrie (Schneidenanzahl $Z$, Durchmesser $D$, Scheidengeometrie). Bei DSST wird die gewählte Spindeldrehzahl anhand des Stabilitätsdiagrammes korrigiert, wenn Ratterfrequenzen während der Bearbeitung festgestellt werden [10]. Dahingegen wird bei der Regelungsstrategie CSSV die Spindeldrehzahl nach Detektieren von Rattern kontinuierlich und periodisch variiert. Besonders eine sinusförmige Variation der Spindeldrehzahl erwies sich zur Vermeidung von niederfrequenten Ratterschwingungen als effizient. Jedoch verursacht die kontinuierliche Variation der Spindeldrehzahl eine ungleichförmige Bauteiloberfläche, die bei bestimmten Anwendungsfällen eine Nachbearbeitung erfordert [9].

Die elegantere Lösung zur Vermeidung von Oberflächenwelligkeiten und hohen

Bild 3. Werkzeugbruchdetektion durch die Überwachung des zu erwartenden und des tatsächlich angreifenden Schnitt-Drehmoments $\tau_{s}$; Anpassung der Überwachungsgrenzen zur Vermeidung von prozessbedingten Fehlbenachrichtigungen auf $\mathrm{Ba}$ sis einer Zeitreihenfilterung und des dazugehörigen

Residuums $\varepsilon_{1}$ [13]
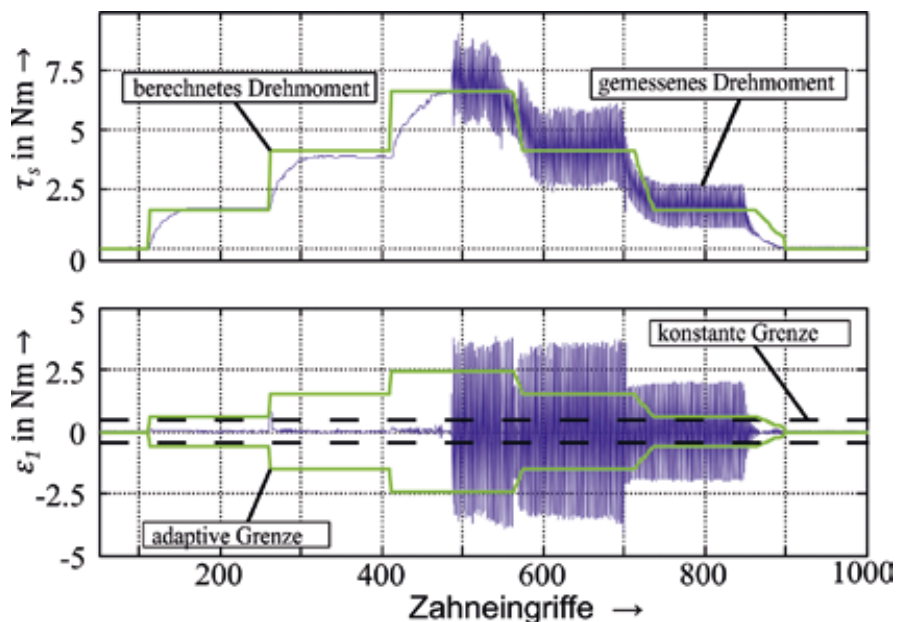

Lasten am Werkzeug und an Vorschubkomponenten ist deshalb, das Auftreten von dynamischen Instabilitäten durch eine günstige, modellunterstützte Prozessparameterauswahl vor Beginn der Bearbeitung auszuschließen. Das strukturdynamische Nachgiebigkeitsverhalten der Werkzeugmaschine, einschließlich des Werkzeuges und des Werkstückes, muss hierfür allerdings bekannt sein.

\section{Werkzeugverschleißüberwachung}

Ein weiterer Schwerpunkt des Projekts „CLM 4.0“ liegt im Bereich der Werkzeugverschleißüberwachung. Die am Werkzeug auftretenden statischen und dynamischen Belastungen beeinflussen die maximal erreichbare Standzeit des Werkzeuges. Aufgrund dessen haben sich Hochleistungswerkstoffe, wie faserverstärkte Kunststoffe und Titan, die vorzugsweise in der Luft- und Raumfahrtindustrie eingesetzt werden, als herausfordernd für die spanende Bearbeitung erwiesen [11]. Die Abnutzungserscheinungen können bei ungünstig gewählten Prozessparametern innerhalb weniger Bearbeitungsschritte so weit fortschreiten, dass die vorgegebenen Form- und Lagetoleranzen sowie die zulässige Oberflächenrauigkeit nicht mehr eingehalten werden können. Infolgedessen muss das finale Bauteil nachgearbeitet oder als Ausschuss eingestuft werden [12]. In beiden Fällen entstehen Kosten, die durch eine zuverlässige Werkzeugüberwachung vermeidbar sind. Im Rahmen des Forschungsprojektes „Closed-Loop-Manufacturing 4.0" wird deshalb eine Online-Verschleißdetektion eingesetzt, die auf einer Überwachung des Spindelmotorstromes basiert. Durch die Kompensation des elektromechanischen Übertragungsver- haltens zwischen Spindelmotor und Bearbeitungszone kann aus der Strommessung das tatsächlich angreifende Prozessmoment bestimmt werden (Bild 3). Des Weiteren bietet eine prozessparallele Berechnung der Zahneingriffsverhältnisse die Möglichkeit, das zu erwartende Drehmoment zu simulieren [13]. Dadurch ist ein sich anpassender Grenzbereich zu bestimmen, der die zulässigen Werte für das Schnitt-Drehmoment $\tau_{s}$ und das dazugehörige Residuum $\varepsilon_{1}$ einer Zeitreihenfilterung vorgibt [6] (Bild 3). Ein plötzlicher Anstieg und ein deutliches Rauschen im Filter-Residuum $\varepsilon_{1}$ lassen auf einen möglichen Schneiden-Bruch schließen. Durch die Signalfilterung und die fortlaufende Adaption der Überwachungsgrenzen werden prozessbedingte Fehlbenachrichtigungen, wie z.B. durch eine sprunghafte Vergrößerung der Zahneingriffsverhältnisse, verhindert [13].

Rekonstruktion der Prozesskräfte zur Bestimmung des positions- und werkzeugabhängigen Nachgiebigkeitsverhaltens

Anhand des Nachgiebigkeitsverhaltens der Struktur kann der zu erwartende Konturfehler aufgrund von quasi-statischer und dynamischer Abdrängung vorhergesagt und reduziert werden. Zusätzlich beeinflusst die Nachgiebigkeit die Stabilität des Bearbeitungsprozess und somit die Ratterneigung entlang der Werkzeugbahnen. Um das strukturdynamische Verhalten von Werkzeugmaschinen $\mathrm{zu}$ bestimmen, wird üblicherweise auf eine experimentelle Modalanalyse mithilfe eines Impulshammers oder eines sog. Shakers (elektromagnetischer Aktuator) zurückgegriffen. Diese Art der Messung ist mit einem hohen manuellen Arbeitsaufwand verbunden, und der Ma- 
a)
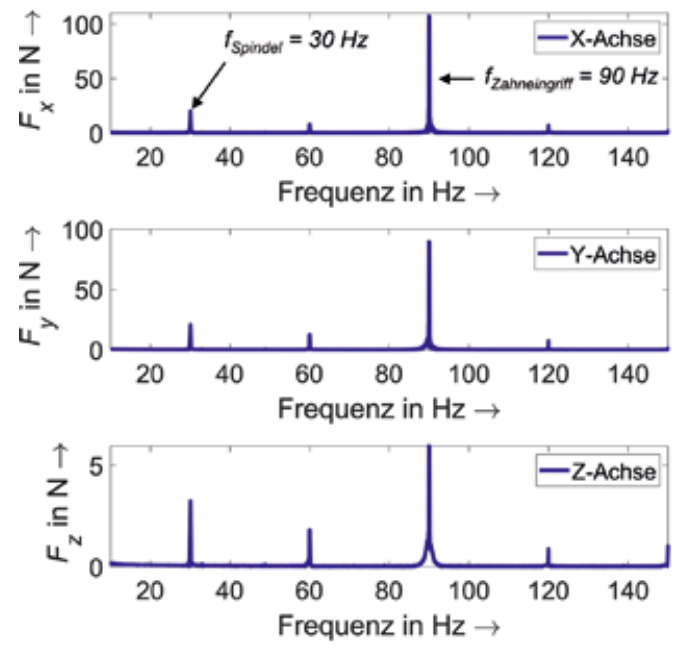

b)
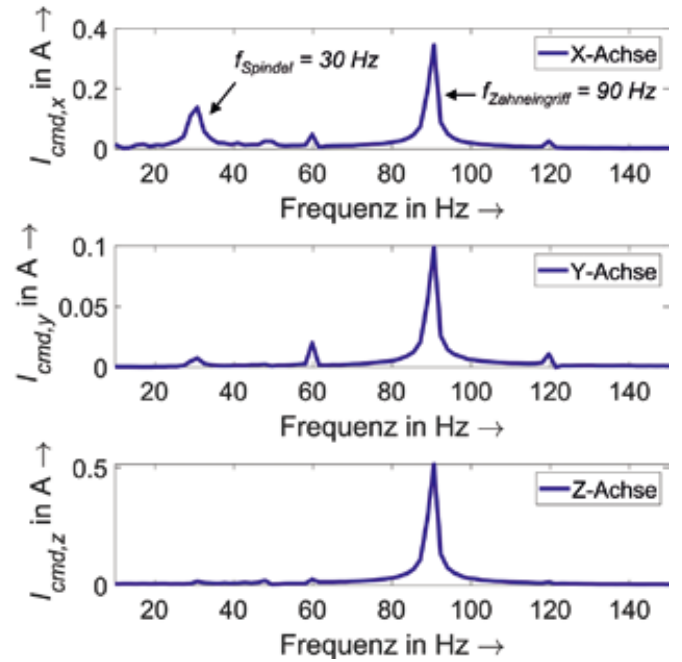

Bild 4. a) Frequenzspektrum der mit einer Kraftmessplattform aufgenommenen Prozesskräfte $F_{x}, F_{y}$ und $F_{z}$ (Datenauflösung $20 \mathrm{kHz}$, Messzeit $10 \mathrm{~s}$ ); b) Frequenzspektrum der Motorströme $I_{c m d, x}, I_{c m d, y}$ und $I_{c m d, z}$ (Datenauflösung $4 \mathrm{kHz}$, Messzeit 0,7 s); Schnittbedingungen: Vollnutenschnitt in X-Richtung; Prozessparameter: Vorschubgeschwindigkeit $v_{f}=450 \mathrm{~mm} / \mathrm{min}$, Spindeldrehzahl $n=1800 \mathrm{U} / \mathrm{min}$, Schnitttiefe $a_{p}=2 \mathrm{~mm}$; Werkstoff: Ck 45; Werkzeug: Mapal OptiMill Uni M3033, Durchmes$\operatorname{ser} D=16 \mathrm{~mm}$, Schneidenanzahl $z=3$, Helix-Winkel $\beta=30^{\circ}$ schinenzustand spiegelt hierbei nicht die tatsächlichen Kraftflussverläufe und Regelungsbedingungen während der Fräsbearbeitung wider. Des Weiteren ist eine kontinuierliche Wiederholung der Modalanalyse während des gesamten Lebenszyklus der Fertigungsanlage notwendig, um auch den Einfluss des Verschleißes der Antriebskomponenten auf die Maschinendynamik feststellen $\mathrm{zu}$ können [14], was mit untragbar hohem Aufwand verbunden ist. Aufgrund dessen ist eine prozessparallele Bestimmung und eine permanente Aktualisierung des positions- und werkzeugabhängigen Nachgiebigkeitsverhaltens ein zentrales Forschungsinteresse des Projekts „Closed-Loop-Manufacturing 4.0“.

Für die prozessparallele Berechnung des Übertragungsverhaltens $H(\omega)$ sind, vergleichbar zur experimentellen Modalanalyse, sowohl die Bestimmung der Anregung $F(\omega)$ als auch die Messung der Frequenzantwort $X(\omega)$ der Maschinenstruktur in Abhängigkeit der Kreisfrequenz $\omega$ notwendig:

$$
H(\omega)=\frac{X(\omega)}{F(\omega)}
$$

Die Strukturanregung basiert dabei auf dem Prozesskraftverlauf, der durch den periodischen Zahneingriff während der Fräsbearbeitung verursacht wird, und ist somit von den Zahneingriffsbedingungen und von den gewählten Fertigungsparametern abhängig. Die Berechnung des Übertragungsverhaltens $H(\omega)$ ist mit sogenannten H-Estimatoren möglich. Die Wahl des Verfahrens hängt davon ab, ob im Anregungs- und Antwortsignal messtechnisches Rauschen vorliegt [14]:
- $H_{1}$ : Das Anregungssignal besitzt Rauschen:

$H_{l}=\frac{S_{f x}(\omega)}{S_{f f}(\omega)}$

- $H_{2}$ : Rauschen liegt nur in der gemessenen Frequenzantwort vor:

$H_{2}=\frac{S_{x x}(\omega)}{S_{x f}(\omega)}$

- $H_{a}$ : Sowohl das Eingangs- als auch das Ausgangssignal weisen Rauschen auf:

$\left|H_{a}\right|^{2}=\frac{S_{x x}(\omega)}{S_{x f}(\omega)}$

Die Bestimmung der Übertragungsfunktion $H(\omega)$ zwischen Anregung $F(\omega)$ und Strukturverhalten $X(\omega)$ kann folglich mithilfe der Kreuzleistungsspektren $S_{x f}$ und $S_{f x}$ und der Autoleistungsspektren $S_{x x}$ und $S_{f f}$ durchgeführt werden. Da das Antwortverhalten mit piezokeramischen Beschleunigungssensoren gemessen wird, ist dort messtechnisches Rauschen nicht $\mathrm{zu}$ vermeiden. Welche Formel zur Berechnung der Übertragungsfunktion zweckmäßigerweise angewendet werden sollte, hängt deshalb von der Bestimmung der Prozesskraft ab.

Im Rahmen des Projekts werden zwei verschiedene Methoden zur Bestimmung der auftretenden Prozesskräfte untersucht und miteinander verglichen. Zum einen lässt die Überwachung der Motorströme der Vorschubachsen einen Rückschluss auf Prozesskraftverläufe und -niveaus zu. Zum anderen können über die Zahneingriffsverhältnisse die Zerspankräfte in den drei unterschiedlichen Raumrichtungen berechnet werden.
Moderne Werkzeugmaschinensteuerungen ermöglichen das Auslesen der kommandierten Soll-Motorströme mit einer Datenauflösung von mehreren Kilohertz. Durch Referenzmessungen mithilfe einer Kraftmessplattform oder eines Impulshammers kann somit das Übertragungsverhalten $G_{F I}$ zwischen den gemessenen Kräften $F$ in der Bearbeitungszone (Bild 4a) und dem StromStellsignal $I_{c m d}$ (vgl. Bild 4b) bestimmt werden. Auf Basis der Inversen dieser Übertragungsfunktion kann ein Kalman-Filter parametriert werden, der wiederum die Rekonstruktion der in der Bearbeitungszone herrschenden Kräfte aus den Motorströmen ermöglicht [13].

Verändert sich das Übertragungsverhalten durch den Austausch der Werkzeug-Spannfutterkombination oder durch den Verschleiß elektromechanischer Komponenten, ist eine erneute Bestimmung des Übertragungsverhaltens und die nochmalige Parametrierung der Kalman-Filterung notwendig. Aufgrund dessen verfolgt das Forschungsvorhaben das Ziel, die Messung des Übertragungsverhaltens $\hat{G}_{F I}$ Zwischen simulierten Prozesskräften und den gemessenen Motorströmen $\mathrm{zu}$ ermöglichen. Für die $\mathrm{Ab}$ schätzung der Zerspankräfte wird die von der Firma ModuleWorks GmbH entwickelte Zahneingriffsberechnung eingesetzt, die auf einer Tri-Dexel-Oberflächenrepräsentation basiert [15]. Eine besondere Herausforderung dabei ist die Synchronisierung von Steuerungsdaten mit der Simulation der Zahneingriffsverhältnisse, aus welchen sich die Zerspankräfte berechnen. 


\section{Prädiktive Instandhaltung von Vorschubantrieben}

Die während der Bearbeitung auftretenden Prozesskräfte verursachen neben Werkzeugverschleiß auch Abnutzungserscheinungen an den Vorschubachsen. Die dort verbauten Kugelgewindetriebe und Profilschienenführungen stellen, wie im ersten Abschnitt erwähnt, die Hauptausfallgruppe von Werkzeugmaschinen dar [16]. Um ungewollte und teure Maschinenausfälle zu vermeiden, verfolgt das von der Europäischen Union geförderte Forschungsprojekt „PreCoM“ einen Ansatz zur Zustandsüberwachung und Restlebensdauerschätzung der Vorschubkomponenten. Dieser basiert auf einer Bestimmung der sogenannten modalen Größen Eigenfrequenz, Eigenvektor und Dämpfung [4]. Üblicherweise werden zur Identifikation dieser Werte externe Beschleunigungssensoren eingesetzt. Die Ausstattung von Produktionsanlagen mit zusätzlicher Sensorik ist jedoch im industriellen Umfeld oft unerwünscht, da höhere Kosten durch Nachrüstungen und weitere Wartungsmaßnahmen die Folge wären. Das $i w b$ verfolgt deshalb das Ziel, eine automatisierte Identifikation der modalen Parameter ausschließlich anhand von CNC-Steuerungsdaten zu realisieren.

Bild 5 zeigt beispielhaft drei verschiedene Nachgiebigkeitsfrequenzgänge der X-Achse einer „DMG duo Block DMC $55 H^{\prime \prime}$, die während eines hierfür entwickelten Testzyklus aufgenommen wurden [4]. Bei der Durchführung der Experimente wurden drei verschiedene Profilschienenführungen mit den unterschiedlichen Vorspannungsklassen C1, C2 und C3 eingesetzt. Die am meisten von den Profilschienenführungen beeinflusste Eigenschwingform (Bild 5a) weist mit abnehmender Vorspannung eine Reduktion der Eigenfrequenz und der dazugehörigen Amplitude auf. Eine kontinuierliche Bestimmung der modalen Parameter, beispielsweise mit dem PolyMAX-Algorithmus [17], ermöglicht es, Veränderungen des dynamischen Verhaltens zu detektieren und somit den aktuellen Verschleißzustand der verschiedenen Vorschubkomponenten zu erfassen.

Darüber hinaus ist es möglich, durch eine intelligente Kombination mit zusätzlichen Maschinendaten, wie z.B. Motorströmen und Positionsdifferenzen, weitere sogenannte verschleißsensitive Merkmale (engl. Features) zu extrahieren [18]. Durch eine probabilistische Klassifikation
Bild 5. Änderungen im dynamischen Verhalten bei einer $A b$ nahme der Vorspannung der Profilschienenführungen; Verwendung verschiedener Vorspannungsklassen

(durchschnittliche Vorspannung $\left.F_{V}\right)$ :

$C_{1}\left(F_{V}=4200 \mathrm{~N}\right)$,

$C_{2}\left(F_{V}=8965 \mathrm{~N}\right)$,

$C_{3}\left(F_{V}=12840 \mathrm{~N}\right)$

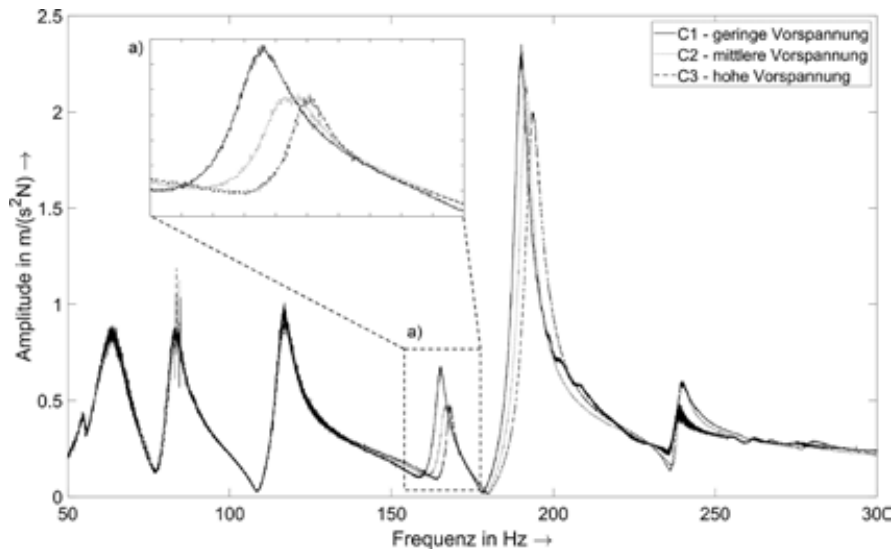

dieser Features kann neben dem aktuellen Verschleißzustand auch dessen zu erwartender Verlauf und damit eine Restlebensdauer abgeschätzt werden. Darauf basierend kann eine prädiktive Instandhaltungsstrategie verfolgt werden, welche Wartungsmaßnahmen rechtzeitig in der Produktionsplanung berücksichtigt [3].

\section{Zusammenfassung}

Dieser Beitrag stellt einen geschlossenen Regelkreis vor, der im Rahmen des kanadisch-deutschen Forschungsprojekts "Closed-Loop-Manufacturing 4.0“ entwickelt wird. Ein Edge-Computer greift über eine Netzwerkkommunikationsarchitektur auf verschiedene Maschinendaten und externe Sensorsignale zu. Auf Basis der akquirierten Daten wird ein maschinennaher (innerer) und ein cloudbasierter (äußerer) Regelkreis ausgeführt (vgl. Bild 1). Der innere Regelkreis übernimmt die zeitkritischen Aufgaben der Rattervermeidung, der Kollisionsüberwachung und der Werkzeugverschleißdetektion. In der äußeren Regelschleife hingegen werden die aufgenommenen Maschinen- und Sensordaten zur Identifikation eines positions- und werkzeugabhängigen Nachgiebigkeitsverhaltens der Maschinenstruktur genützt. Dadurch soll unter anderem sichergestellt werden, dass keine dynamischen Instabilitäten während der Zerspanung auftreten und die Toleranzen für Konturfehler sowie die maximal zulässigen Bearbeitungskräfte nicht überschritten werden. Des Weiteren zeigt dieser Beitrag, wie durch eine wiederholte Aufnahme des dynamischen Verhaltens der aktuelle Verschleißzustand der Vorschubantriebe detektiert werden kann. Eine Kombination mit zusätzlichen Maschinendaten ermöglicht es, weitere verschleißsensitive Merkma- le zu extrahieren, welche die Vorhersage der Restlebensdauer von Kugelgewindetrieben und Profilschienenführungen erlauben. Das EU-Projekt „PreCoM“ greift auf diese Restlebensdauerschätzung zurück, um vorausschauend Wartungsmaßnahmen in der Produktionsplanung zu berücksichtigen und ungeplante Stillstandzeiten zu vermeiden.

\section{Literatur}

1. Milberg, J.: Werkzeugmaschinen - Grundlagen. Zerspantechnik, Dynamik, Baugruppen und Steuerungen. Springer-Verlag, Berlin, Heidelberg 1995

2. Weck, M.; Brecher, C.: Messtechnische Untersuchung und Beurteilung, dynamische Stabilität. Werkzeugmaschinen (Bd. 5). Springer-Verlag, Berlin, Heidelberg 2006

3. Zhai, S; Reinhart, G.: Predictive Maintenance als Wegbereiter für die instandhaltungsgerechte Produktionssteuerung. ZWF 113 (2018) 5, S. 298-301 DOI: $10.3139 / 104.111912$

4. Ellinger, J. et al.: Feed Drive Condition Monitoring Using Modal Parameters. MM Science Journal 2019 (2019) 4, S. 3206-3213 DOI: 10.17973/MMSJ.2019_11_2019072

5. Tseng, G.W.G.; Chen, C. Q. G.; Erkorkmaz, K.; Engin, S.: Digital Shadow Identification from Feed Frive Structures for Virtual Process Planning. CIRP Journal of Manufacturing Science and Technology 24 (2019), S. 55-65 DOI: 10.1016/j.cirpj.2018.11.002

6. Altintas, Y.: Manufacturing Automation Metal Cutting Mechanics, Machine Tool Vibrations, and CNC Design. Cambridge University Press, Cambridge 2012

7. Yoneoka, T.; Kakinuma, Y.; Ohnishi, K. Aoyama, T.: Disturbance Observer-Based Inprocess Detection and Suppression of Chatter Vibration. Procedia CIRP 1 (2012), S. 44-49 DOI: 10.1016/j.procir.2012.04.006

8. Kleinwort, R.; Weishaupt, P.; Zaeh, M. F.: Simulation-Based Dimensioning of the Required Actuator Force for Active Vibration Control. International Journal of Automation Technology 12 (2018) 5, S. 658-668 DOI: 10.20965/ijat.2018.p0658 
9. Munoa, J.; Beudaert, X.; Dombovari, Z.; Altintas, Y.; Budak, E.; Brecher, C.; Stepan, G.: Chatter Suppression Techniques in Metal Cutting. CIRP Annals 65 (2016) 2, S. 785-808 DOI: 10.1016/j.cirp.2016.06.004

10. Friedrich, J.; Torzewski, J.; Verl, A.: Online Learning of Stability Lobe Diagrams in Milling. Procedia CIRP 67 (2018), S. 278-283 DOI: 10.1016/j.procir.2017.12.213

11. M'Saoubi, R.; Axinte, D.; Soo, S. L.; Nobel, C.; Attia, H.; Kappmeyer, G.; Engin, S.;

Sim, W.-M.: High Performance Cutting of Advanced Aerospace Alloys and Composite Materials. CIRP Annals 64 (2015) 2, S. 557-580 DOI: 10.1016/j.cirp.2015.05.002

12. Griffin, R.; Cao, Y.; Peng, J. Y.; Chen, X. B.: Tool Wear Monitoring and Replacement for Tubesheet Drilling. International Journal of Advanced Manufacturing Technology 86 (2016) 5-8, S. 2011-2020 DOI: $10.1007 / \mathrm{s} 00170-015-8325-2$

13. Altintas, Y.; Aslan, D.: Integration of Virtual and Online Machining Process Control and Monitoring. CIRP Annals 66 (2017) 1, S. 349-352 DOI: 10.1016/j.cirp.2017.04.047

14. Cai, H.; Mao, X.; Li, B.; Luo, B.: Estimation of FRFs of Machine Tools in Output-only Modal Analysis. International Journal of Advanced Manufacturing Technology 77 (2015) 1-4, S. $117-130$ DOI: $10.1007 / \mathrm{s} 00170-014-6439-6$

15. Katz, A.; Erkorkmaz, K.; Ismail, F.: Virtual Model of Gear Shaping - Part I: Kinematics, Cutter - Workpiece Engagement, and Cutting Forces. Journal of Manufacturing Science and Engineering 140 (2018) 7, S. 26 DOI: $10.1115 / 1.4039646$
16. Fleischer J.; Schopp M.; Broos A.; Wieser J.: Datenbasis für lastabhängige Prozesseingriffe. Modularisierung und Analyse von Ausfallursachen zur Erhöhung der Verfügbarkeit von Werkzeugmaschinen. wt Werkstattstechnik online 97 (2007) 7, S. 491-497

17. Phillips, A.W.; Allemang, R.J.; Brown, D. L.: Autonomous Modal Parameter Estimation: Methodology 3 (2011), S. 363-384 DOI: 10.1007/978-1-4419-9299-4 32

18. Benker, M.; Kleinwort, R.; Zäh, M.F.: Estimating Remaining Useful Life of Machine Tool Ball Screws via Probabilistic Classification. In: Proceedings of the 2019 IEEE International Conference on Prognostics and Health Management (ICPHM). San FrancisCo, CA, USA 2019, S. 1 - 7 DOI: 10.1109/ICPHM.2019.8819445

\section{| Die Autoren dieses Beitrages}

Benedikt Schmucker, M.Sc., geb. 1992, studierte Luft- und Raumfahrt und Maschinenbau an der Technischen Universität München (TUM) und ist Wissenschaftlicher Mitarbeiter am Institut für Werkzeugmaschinen und Betriebswissenschaften $(i w b)$

Johannes Ellinger, M. Sc., geb. 1990, studierte Maschinenbau an der TUM und ist Wissenschaftlicher Mitarbeiter am iwb.

Maximilian Benker, M. Sc., geb. 1990, studierte Wirtschaftsingenieurwesen an der Technischen Universität Berlin und ist wissenschaftlicher Mitarbeiter am $i w b$.

Robin Kleinwort, M. Sc., geb. 1988, studierte Maschinenbau an der TUM und ist ehemaliger Leiter der Themengruppe Werkzeugmaschinen am $i w b$.
Thomas Semm, M. Sc., geb. 1991, studierte Maschinenbau an der TUM und leitet die Themengruppe Werkzeugmaschinen am iwb.

Prof. Dr.-Ing. Michael F. Zäh, geb. 1963, leitet seit 2002 gemeinsam mit Prof. Dr. Ing. Gunther Reinhart das $i w b$ der TUM.

\section{Summary}

The economic use of machine tools is highly dependent on the material removal rate and the amount of machine downtime. As a result, manufacturing companies focus on increasing feed rates and cutting depths and on reducing the number of necessary maintenance measures. The digital twin enables the optimization of machining processes, while obeying the static and dynamic load limits, through a permanent data exchange between the real machine tool and its virtual representation. Additionally, the acquisition of data during the lifetime of machine tools allows to detect changes in the dynamic behaviour of the feed drive components. Variations of the determined modal parameters indicate changes in the wear condition. By means of a probabilistic classification its future progression can be forecasted.

\section{Bibliography}

DOI 10.3139/104.112303

ZWF 115 (2020) Special; page 78-83

(C) Carl Hanser Verlag GmbH \& Co. KG ISSN 0947-0085 